\title{
The filamin-B-refilin axis - spatiotemporal regulators of the actin-cytoskeleton in development and disease
}

\author{
Jacques Baudier ${ }^{1,2, *}$, Zandra A. Jenkins ${ }^{3}$ and Stephen P. Robertson ${ }^{3, *}$
}

\begin{abstract}
During development, cycles of spatiotemporal remodeling of higherorder networks of actin filaments contribute to control cell fate specification and differentiation. Programs for controlling these dynamics are hard-wired into actin-regulatory proteins. The filamin family of actin-binding proteins exert crucial mechanotransduction and signaling functions in tissue morphogenesis. Filamin-B (FLNB) is a key player in chondrocyte progenitor differentiation for endochondral ossification. Biallelic loss-of-function mutations or gain-of-function mutations in FLNB cause two groups of skeletal disorders that can be attributed to either the loss of repressive function on TGF- $\beta$ signaling or a disruption in mechanosensory properties, respectively. In this Review, we highlight a unique family of vertebrate-specific short-lived filamin-binding proteins, the refilins (refilin-A and refilin-B), that modulate filamin-dependent actin crosslinking properties. Refilins are downstream TGF- $\beta$ effectors in epithelial cells. Double knockout of both refilin-A and refilin-B in mice results in precocious ossification of some axial skeletal elements, leading to malformations that are similar to those seen in FLNBdeficient mice. Based on these findings, we present a model summarizing the role of refilins in regulating the mechanosensory functions of FLNB during skeletal development. We also discuss the possible contribution of refilins to FLNB-related skeletal pathologies that are associated with gain-of-function mutations.
\end{abstract}

KEY WORDS: Refilin-A, Refilin-B, FAM101A, FAM101B, Filamin, Actin, TGF- $\beta$, Epithelial-mesenchymal transition, Filaminopathy

\section{Introduction}

During development, cell fate specification and differentiation is coupled to the cellular acquisition of specific adhesive, polar and motile behaviors. Various higher-order networks of actin filaments and spatiotemporal cytoskeletal dynamics are used to organize cellcell contacts and cell-substratum adhesion, cell membrane remodeling and to power cell motility (Davidson and Wood, 2016). A myriad of actin-binding proteins participate in the formation of actin filaments by regulating actin polymerization (reviewed in Davidson and Wood, 2016) and the depolymerization of actin filaments (Nag et al., 2013; Umeki et al., 2013). Further studies have also progressed our understanding of the role of mechanosensing actin-binding proteins in the control of spatiotemporal actin dynamics in developmental processes

\footnotetext{
${ }^{1}$ Aix Marseille Université, CNRS, IBDM, 13284 Marseille Cedex 07, France. ${ }^{2}$ Institu de Biologie du Développement de Marseille-UMR CNRS 7288, Campus de Luminy-Case 907, 13288 Marseille Cedex 9, France. ${ }^{3}$ Department of Women's and Children's Health, Dunedin School of Medicine, University of Otago, Dunedin, New Zealand.

*Authors for correspondence (jacques.BAUDIER@univ-amu.fr; stephen.robertson@otago.ac.nz)
}

(D) J.B., 0000-0001-8427-4444
(Huelsmann et al., 2016; Janoštiak et al., 2014). These proteins are able to sense and respond to forces, for instance, by undergoing conformational changes (Vogel et al., 2001), which can expose cryptic binding sites (Pentikäinen and Ylanne, 2009), or even by becoming more tightly bound to one another (Schoeler et al., 2014). Filamins have been shown to be a central mechanotransduction element of the actin cytoskeleton (Ehrlicher et al., 2011; Huelsmann et al., 2016; Janoštiak et al., 2014; Luo et al., 2013; Razinia et al., 2012; Seppälä et al., 2017; Stossel et al., 2001) and, in addition, exert signaling functions (Razinia et al., 2012; Stossel et al., 2001; Zhou et al., 2010). Three filamin-encoding genes are found in humans: the X-chromosomal FLNA, and autosomal FLNB and $F L N C$. These genes encode their respective proteins filamin-A (FLNA), filamin-B (FLNB) and filamin-C (FLNC), all of which share a high sequence similarity. Various mutations that give rise to various developmental syndromes have been characterized in all three filamin genes (Bicknell et al., 2007; Clark et al., 2009; Fürst et al., 2013; Robertson et al., 2006, 2003). For instance, mutations in FLNB cause a group of chondrodysplasias, an observation that is consistent with the expression of this protein in epiphyseal growth plate chondrocytes (Krakow et al., 2004). Two broad groups of FLNB-related skeletal conditions can be defined on the basis of their clinical presentation and genetic etiology. Homozygosity or compound heterozygosity for null alleles results in the recessive condition spondylocarpotarsal syndrome (SCTS), which features fusion of the vertebral, carpal and tarsal bones (Krakow et al., 2004). In mice, disruption of Flnb leads to abnormal differentiation of intervertebral disk chondrocytes into hypertrophic chondrocytes, resulting in progressive vertebral fusions. The phenotype observed for Flnb-null mice is consistent with the clinical features of SCTS (Farrington-Rock et al., 2008; Zheng et al., 2007; Zhou et al., 2007; Zieba et al., 2016). This phenotype coincides with an upregulation of TGF- $\beta$ signaling via Smad2 and Smad3 (Zheng et al., 2007; Zieba et al., 2016). A second group of autosomal dominant diseases is caused by missense mutations or small in-frame deletions or insertions in FLNB. These phenotypes include boomerang dysplasia (BD), Larsen syndrome (LS), and atelosteogenesis types I and III (AOI and AOIII) (Xu et al., 2017). In the more-severe forms of these disorders, bones are either shortened or abnormally shaped due to anomalous ossification, or have failed to initiate ossification altogether (Bicknell et al., 2007); further details regarding the disease conditions can be found in $\mathrm{Xu}$ et al. (2017). When ectopically expressed in cells, mutant FLNB with BD, AO or LS-associated mutations showed enhanced avidity for binding actin, resulting in the formation of abnormal foci containing FLNB-actin superstructures, suggesting that these diseases might be caused by the disruption of the mechanosensory properties of filamin (Bicknell et al., 2007; Clark et al., 2009; Daniel et al., 2012; Zhao et al., 2016).

To further expand the understanding of filamin functions during skeletogenesis in physiological and pathological situations, it is 
necessary to comprehensively understand the interplay between the many filamin-binding, scaffolding and signaling proteins that link these proteins to specific spatio-temporal developmental processes, including the effects produced through mechanosensory mechanisms. Recent studies have identified a new family of vertebrate-specific filamin-binding proteins, comprising refilin-A and refilin-B, that fulfill the criteria as spatiotemporal regulators of the mechanosensory functions of filamin during vertebrate skeletal development. Refilins are short-lived proteins and downstream effectors of TGF- $\beta$ signaling (Gay et al., 2011a). In cells, refilins integrate the mechanosensory functions of filamin by modulating their crosslinking properties, so that actin bundles are favored over orthogonally crosslinked networks (Gay et al., 2011a,c). Although the phenotypes of single knockouts (KOs) of refilin-A and refilin-B are indistinguishable from wild-type, double $\mathrm{KO}$ (DKO) mice display skeletal abnormalities that are similar to the malformations observed in Flnb-deficient mice (Mizuhashi et al., 2014). DKO mice for refilin-A and refilin-B manifested scoliosis and kyphosis (pathological curvatures of the spine in the sagittal and transverse planes, respectively), intervertebral disc defects, vertebral fusions and retardation of longitudinal bone growth (Mizuhashi et al., 2014). The skeletal abnormalities reported for $R f \ln$ DKO mice are similar to the malformations in Flnb-deficient mice, which also manifest scoliosis, kyphosis and vertebral fusions, in addition to shortening of the distal appendages (Farrington-Rock et al., 2008; Zheng et al., 2007; Zhou et al., 2007). In humans, a drastic decrease of the expression level of the refilin-A gene (RFLNA, also known as FAM101A) is observed in adolescent idiopathic scoliosis (AIS), a human disease resulting in a three-dimensional deformity of alignment of the spine in the context of an otherwise stucturally normal vertebral column (Fendri et al., 2013), further suggesting that refilins mediate key functions in spinal development and growth. Furthermore, upregulation of refilin-B is observed in metastatic cancer cells (Gordon et al., 2017; Mishra et al., 2015).

This Review synthesizes our current understanding of the functional properties of the refilin family of proteins (refilin-A and refilin-B) as downstream effectors in TGF- $\beta$ signaling and in regulating the mechanosensory functions of FLNB, with special emphasis on skeletal development and in FLNB-related pathologies.

\section{The vertebrate filamins}

The filamin family of actin-binding proteins was first identified as an actin-binding protein (ABP) that binds immunoglobulin (Ig) $\mathrm{G}$ Fc receptor I (Ohta et al., 1991). The filamin proteins are represented in nearly all metazoa (Light et al., 2012). Filamins can crosslink actin filaments into orthogonal networks in the cortical cytoplasm and participate in the anchoring of membrane proteins to the actin cytoskeleton (Calderwood et al., 2001; Nakamura et al., 2007, 2011; Razinia et al., 2012). By linking actin to the extracellular matrix (ECM), filamins can simultaneously bind to actin and the cytoplasmic domains of transmembrane receptors (Baldassarre et al., 2009; Razinia et al., 2012; Sasaki et al., 2001). Through rapid and transient modulation of the interactions between the actin cytoskeleton and the cell membrane or ECM, filamins enable spatiotemporal changes in the transmission of forces at the cell periphery and, as such, serve as mechanotransducers (Huelsmann et al., 2016; Razinia et al., 2012). In addition to actin, filamins interact with more than 90 binding partners and are implicated in signaling for a diverse array of cellular functions, including motility and differentiation (Razinia et al., 2012; Zhou et al., 2010).

Phylogenetically, filamins diverged from a common single ancestral gene between the chordate invertebrate and vertebrate lineages (Kesner et al., 2010). A single filamin gene is present in the Drosophila melanogaster genome (cheerio), that encodes two spliced protein isoforms (Sokol and Cooley, 1999), whereas two genes ( $f l n-1$ and $f l n-2)$ exist in the Caenorhabditis elegans genome. In Drosophila, Cheerio is involved in the germline ring canal formation where it tethers actin filaments within the ovarian ring canal to the cell membrane (Sokol and Cooley, 1999). In C. elegans, FLN-1 acts as a stretch-sensitive signaling scaffolder to control valve dilation and the exit of oocytes from the spermatheca (DeMaso et al., 2011; Kovacevic and Cram, 2010). In vertebrates, the filamin gene has been duplicated twice, generating three genes localized on three different chromosomes: FLNA (Xq28), FLNB (3p14) and FLNC (7q32) (Zhou et al., 2010). These genes encode their respective proteins filamin-A (FLNA), filamin-B (FLNB) and filamin-C (FLNC), all of which show high sequence similarity and similar functional organization (Razinia et al., 2012; Zhou et al., 2010) (Fig. 1A). Mouse knockout models for filamins indicate that vertebrate filamins are involved in three-dimensional tissue morphogenesis and are critical for skeletal, vascular, muscular, cardiac and cerebral development (Dalkilic et al., 2006; Feng et al., 2006; Robertson, 2005; Sheen et al., 2002; Zhou et al., 2010).

Although invertebrate and vertebrate filamins are very similar, differences also exist (Huelsmann et al., 2016; Kesner et al., 2010; Light et al., 2012). Sequence alignment shows that Ig repeats 7-11 are missing within the N-terminal half of the invertebrate filamins (Fig. S1). Moreover, the filamin Ig domain architecture differs substantially between vertebrates and invertebrates, suggesting a specialized function for vertebrate filamins that is linked to the binding of specific signaling molecules (Light et al., 2012). In vertebrate filamin, Ig-repeats 9-15 contain an F-actin-binding domain necessary for high avidity F-actin binding (Nakamura et al., 2007; Zhou et al., 2010). As discussed below (Fig. 1B), the Ig repeats 9-15 might contribute to refilin binding and to the actinbundling function of filamin proteins.

\section{The refilin family of genes and proteins}

The refilin family of genes (Rfln; HUGO Gene Nomenclature Committee, NCBI Gene ID: 144347 and 359845) was first identified by expression screening of a cDNA library of embryonic day (E)8.0-8.5 mouse anterior neuroectoderm to identify genes that function in regionalization of this structure (Hirano et al., 2005). Rfln genes are confined to the vertebrate phylum and there are no homologs in the genomes of Drosophila or C. elegans.

In vertebrates, two refilin paralogs exist, $R f \ln A$ (also known as $C f m 2$ and Fam101a) and RflnB (also known as Cfml and Fam101b), which encode refilin-A and refilin-B, respectively, and were named because of their ability to bind and regulate of filamin proteins (REgulator of Filamin proteIN) (Gay et al., 2011a). According to the HUGO Gene Nomenclature Committee, human RFLNA localizes to chromosome 12, whereas RFLNB is on chromosome 17. Gene expression analysis and regulation, as annotated in the GeneCards database (http://www.genecards.org) show that both $R f \ln A$ and $R f \ln B$ harbor common regulatory elements in their promoter regions for binding the PKNOX1 (also known as PREP1) transcription factor involved in the regulation of embryonic development and in the determination of cell fate (Yoshioka et al., 2015). Remarkably, both refilin-B and PKNOX1 are modulators of cell responsiveness to TGF- $\beta$ and are necessary for the induction of the epithelial-mesenchymal transition (EMT) in response to this growth factor (Gay et al., 2011a; Risolino et al., 2014). 
A

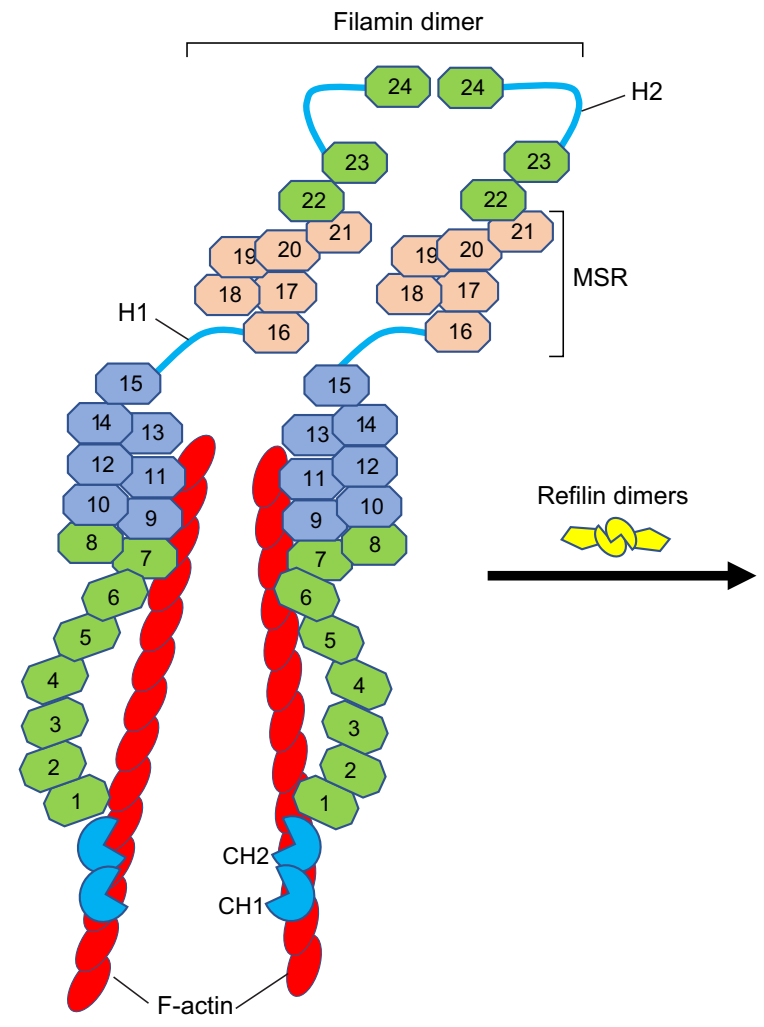

B

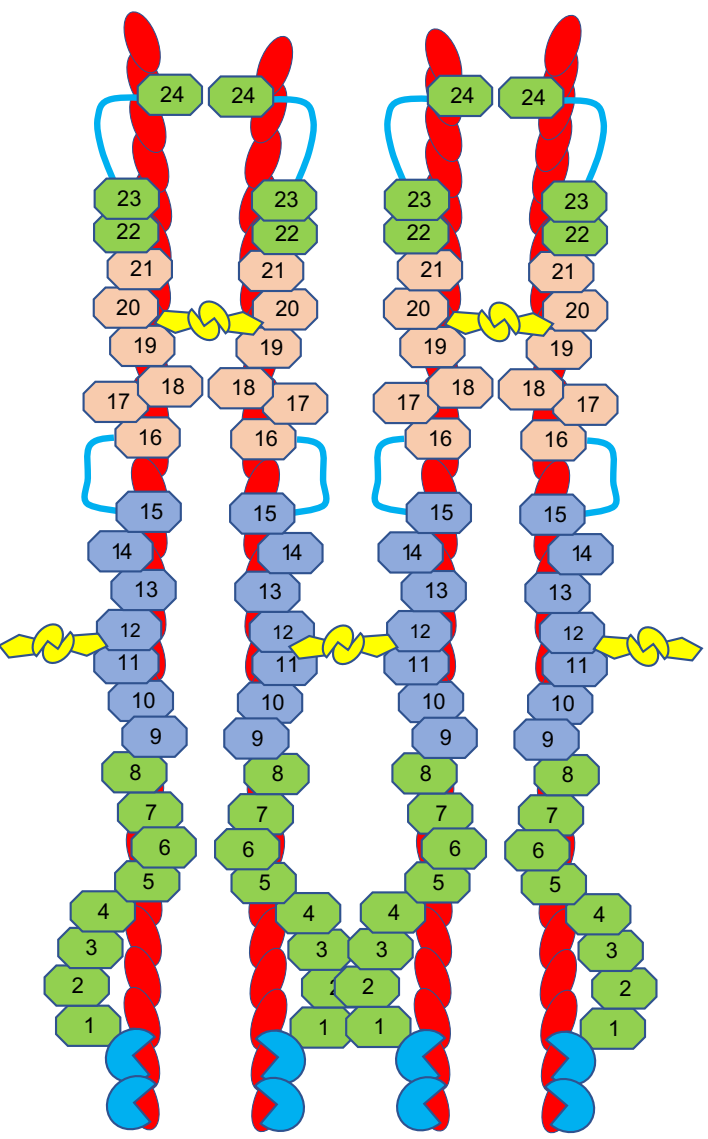

Fig. 1. Proposed model for how refilin binding to filamin induces conformational changes in filamin and promotes the bundling of F-actin filaments. (A) A schematic of a vertebrate filamin dimer. Vertebrate filamins are elongated dimeric $V$-shaped proteins with two large polypeptide chains. Each monomeric chain of filamins contains two calponin homology domains $(\mathrm{CH} 1$ and $\mathrm{CH} 2)$ forming a canonical F-actin-binding domain (bright blue) at the $\mathrm{N}$-terminus. The $\mathrm{ABD}$ is followed by $24 \beta$-pleated sheet immunoglobulin (Ig)-like repeats (numbered octagons), which are interrupted by two flexible hinge regions (H1 and $\mathrm{H} 2$ ). The last C-terminal Ig repeat (Ig24) serves as a dimerization domain. In both vertebrate and invertebrate filamins, the C-terminal domain (Ig16-21 in vertebrates) functions as a mechanosensor region (MSR, pink). It has two protein-interaction sites that are masked by neighboring sequences in an auto-inhibitory manner (closed conformation), with unmasking resulting from pulling forces (open conformation) (Huelsmann et al., 2016; Rognoni et al., 2012). The cryptic F-actin-binding domain IgFLN9-15 is in light blue (Nakamura et al., 2007; Zhou et al., 2010). The dimeric structure of FLNA favors orthogonal junctions of actin filaments and anchors F-actin to the cell membrane through transmembrane receptors (Razinia et al., 2012; Zhou et al., 2010). (B) Schematic illustration of the filamin-refilin complex. The binding of refilin dimer to IgFLNA19-21 may lock filamin into an open conformation (Huelsmann et al., 2016). The subsequent interaction of the refilin dimer with IgFLN10-12 exposes the cryptic F-actin-binding domain on IgFLN9-15, which is necessary for high-avidity F-actin binding (Nakamura et al., 2007; Zhou et al., 2010). This may change the orientation of the FLNA rod regions (Rod 1-lgFLNA1-15 and Rod 2-lgFLNA16-24) and convert the V-shaped FLNA dimer into a low-angle dimer that is more predisposed for actin bundling (Gay et al., 2011a; Gay et al., 2011c). This model also suggests that refilin dimers stabilize the actin filament. It also raises the possibility that refilin dimers bound to filamin IgFLN10-12 could further cross-link F-actin bundles to promote formation of large actin superstructures as observed in the human U373A cells characterized by deficient refilin degradation pathways (see fig. S2 and S3 in Gay et al., 2011a).

The two $R f \ln A$ and $R f \ln B$ paralogs code for proteins with more than $70 \%$ amino acid identity and apparently redundant function. Mice with loss of a single Rfln gene demonstrate no ostensible abnormalities, whereas only double homozygotes for deletion of both $R f \ln A$ and $R f \ln B$ manifest skeletal malformations as mentioned above (Mizuhashi et al., 2014). Refilins have two functional conserved domains (Hirano et al., 2005) (Fig. 2A). The conserved $\mathrm{N}$-terminal domain of refilin functions as a dimerization interface, whereas the C-terminal domain harbors the filamin-binding sites (Gay et al., 2011a).

The short half-life of refilins makes the endogenously expressed proteins difficult to detect by conventional biochemical and immunological approaches, unless the proteins are stabilized by environmental cues, such as cell confluence (Gay et al., 2011a,b) or treatment of cells with TGF- $\beta$ (Gay et al., 2011a). The short half-life of the refilins is related to them being mostly unstructured (Fig. 2B) and the presence of several destruction motifs (Gay et al., 2016). In cells, the stability of intrinsically unstructured proteins (IUPs) is finely tuned so they persist in an appropriate stoichiometry with their physiological targets (Dyson and Wright, 2005; Gsponer et al., 2008). In the case of refilins, their stabilization depends on interaction with their target protein, that is, filamin. In the human M2 cell line that does not express filamin, ectopically expressed rat refilin-A and refilin-B are immediately degraded (Gay et al., 2016). In this context, co-expression with filamin or filamin minigenes encompassing their refilin-binding domains, results in stabilization of refilins such that they have a half-life of between $30 \mathrm{~min}$ and $4 \mathrm{~h}$ depending on the cell culture density (Gay et al., 2016).

When refilin is bound to filamin, its degradation relies on complex degradation pathways that recognize several destruction motifs within the N-terminal and C-terminal domains (Gay et al., 2016). The N-terminal dimerization domain of refilins harbors two 
A

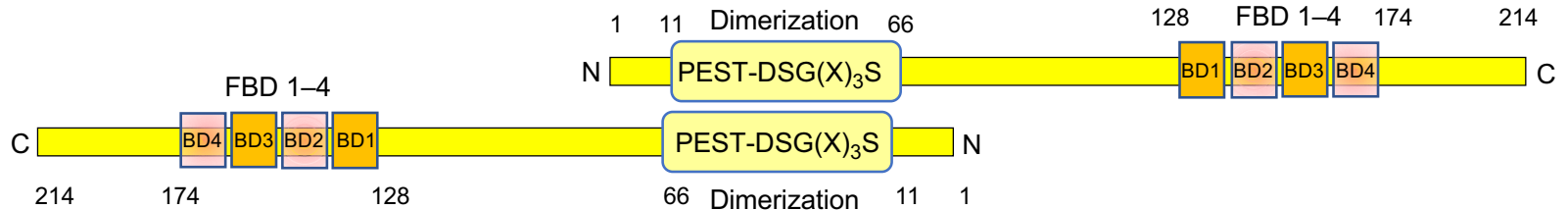

B

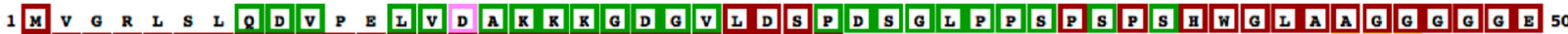

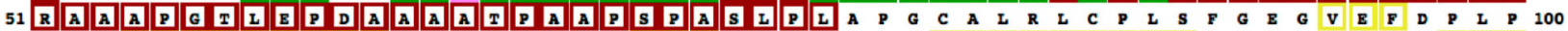

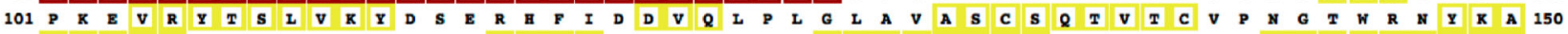

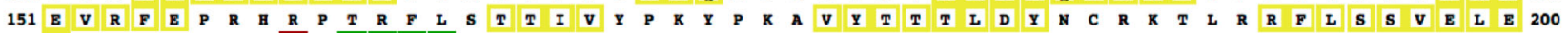
201 A

Key

M Helix L Sheet $\mathrm{E}$ Disordered E Disordered protein binding

Fig. 2. The refilin proteins. (A) A schematic illustration of the refilin-B dimer. Refilin-A and refilin-B are small hydrophilic proteins with more than $70 \%$ amino acid identity (human refilin-A, 216 amino acids; human refilin-B, 214 amino acids). The N-terminal domain of refilins, encompassing residues 10-66, has been defined as a dimerization interface by yeast two-hybrid-directed matings and co-immunoprecipitation (Gay et al., 2011a). It also harbors two overlapping degradation signals (residues 10-35) consisting of a PEST sequence and a phosphodegron motif $\left[\mathrm{DSG}(\mathrm{X})_{3} \mathrm{~S}\right]$. The C-terminus harbors the filamin-binding domain (FBD), which comprise four individual interaction sites (BD1-BD4) and is also a target for the rapid degradation of the protein (see text for further details). (B) Secondary structure map of human refilin-B. PSI-blast-based secondary structure PREDiction (http://bioinf.cs.ucl.ac.uk/psipred/) (PSIPRED) reveals that refilins are intrinsically unstructured proteins (IUPs), with nearly $50 \%$ unstructured residues and no predicted $\alpha$-helical domains. Feature predictions are colorcoded in the sequence according to the key shown below. Disordered protein binding regions may also putatively constitute protein binding motifs.

overlapping degradation signals (degrons), consisting of a PEST sequence and a DSG(X) $)_{3}$ S motif (Gay et al., 2011b). The DSG(X) $)_{3} S$ motif is commonly called a phosphodegron (phosphorylationdependent degron motif), because phosphorylation of serine residues within the degron is required to activate it (Ang and Wade Harper, 2005). Selective removal of the PEST-DSG $(\mathrm{X})_{3} \mathrm{~S}$ motif (residues 10-35) from the rat refilin-A increased the half-life of the truncated proteins. However, the truncated refilin-A protein was still subjected to fast degradation processes (Gay et al., 2016). A third degron has then been localized within the $\mathrm{C}$-terminus of the protein that is targeted by a proteasome-dependent (MG132 sensitive) but ubiquitin-independent pathway. This $\mathrm{C}$-terminal degron overlaps with the filamin-binding motif on refilin (residues $128-175$ on refilin-A), as its activity can be replicated by GFP-tagged refilin-A 128-175. Attaching the 128-175 region of refilin to GFP resulted in rapid degradation of the GFP moiety compared to non-heterologous GFP (see fig. S3 in Gay et al., 2016). The complexity of mechanisms governing the stabilization and the degradation of the refilins is reminiscent of that of many short-lived cell-cycle-regulated proteins (e.g. IкB $\alpha, \mathrm{Cdc} 25 \mathrm{~A}$ ), transcription factors (TAZ, Nrf1) and receptors (EPO-R) that are targeted by several independent, but complementary, proteolytic pathways (Fortmann et al., 2015; Huang et al., 2012; Kanemori et al., 2005; Tsuchiya et al., 2011; Walrafen et al., 2005). Therefore, the comprehensive characterization of how the stability and abundance of refilin mRNA and protein are determined is a priority. The modulation of refilin turnover in cells is likely to have a crucial function in the spatiotemporal regulation of the mechanosensory functions of filamin. Thus far, refilins are the only described filamin-binding partners that modulate its actinbranching properties so that actin bundling is favored (Gay et al., 2011a; Mizuhashi et al., 2014). The actin-bundling activity that is mediated by refilins is likely to produce changes not only in actin cystokeletal organization, but also in the interaction of filamin with other partners (e.g. transmembrane receptors, cell adhesion molecules and signaling molecules) (Razinia et al., 2012; Stossel et al., 2001; Zhou et al., 2010). Hence, the interactions between refilins and filamins must be fully reversible to facilitate these rapidly fluctuating cytoskeletal dynamics.

\section{Role of refilin in filamin-mediated actin bundling}

In both a reconstituted in vitro actin polymerization system and in vivo, the binding of refilins to filamin converts filamin from a protein that mediates actin-branching into one that mediates bundling (Gay et al., 2011a). In vitro, our electron microscopy data has revealed the presence of large F-actin bundles even at a low ratio of FLNA to actin (1:100). Similarly, ectopic expression of refilins in mesenchymal U373 cells promotes the re-localization of FLNA from a diffuse cytoplasmic staining to newly formed actin bundles that organize either as a perinuclear actin cap or basal stress fibers connected to focal adhesion sites (Gay et al., 2011a,c). At the tips of focal adhesions, refilin, but not FLNA, is excluded from the actin cytoskeleton that is connected to ECM adhesion complexes (J.B., unpublished observations).

Characterization of the refilin-A-FLNA interaction revealed the presence of four potential filamin-binding domains (BD1-BD4) on the refilin monomer, which are clustered on its C-terminus (Fig. 2A). Two of these domains, BD2 and BD4, are essential for co-immunoprecipitation of refilin-A with IgFLNA19-22 and for actin-bundling activity in vitro and in vivo (Gay et al., 2011a). BD1 and BD3 are dispensable for interaction of refilin-A with IgFLNA19-22, but they are required to promote multimolecular refilin-FLNA complexes and actin-bundling activities (see fig. 3F in Gay et al., 2011a). Taken together, these results suggest the presence of two refilin-binding sites on FLNA. By using a directed yeast two-hybrid mating system for refilin-A (amino acids 11-216) with truncated C-terminal FLNA variants, we confirmed that IgFLNA21 serves as a template for the interaction of refilin BD2 and BD4 with FLNA monomers (Gay et al., 2011c). We have now additional evidence that also implicates IgFLNA11-12 in the binding to refilins (Z.A.J. and S.P.R., unpublished data). In Fig. 1B, we propose a tentative model for the refilin-filamin interaction and refilin-dependent modulation of filamin-actin crosslinking properties. The binding of refilin BD2 and BD4 to IgFLNA19-21 may lock filamin into an open conformation (Huelsmann et al., 2016). The subsequent interaction of refilin BD1 and BD3 with IgFLN10-12 activates the cryptic F-actin-binding domain on IgFLN9-15 that is necessary for high-avidity F-actin binding 
(Nakamura et al., 2007), thereby synergizing with the actin-binding domain to promote the formation of F-actin bundles. We also propose that through the resulting increased affinity of filamin for F-actin filaments, refilins may enhance filament stability and growth (Kueh et al., 2008).

\section{Refilins initiate filamin-dependent spatiotemporal changes in apical F-actin}

Several observations point to a spatiotemporal regulation of the apical actin cytoskeletal by refilin-filamin complexes in mechanosensing processes, which may have fundamental roles during vertebrate organogenesis.

In exponentially growing mouse NIH3T3 fibroblasts, the steadystate level of refilin-B is extremely low and rises drastically as the cells reach confluence, suggesting that refilin-B expression and/or stabilization is linked to the cellular response to mechanical force. In confluent fibroblast cells, refilin-B forms complexes with filamin and promotes the formation of a perinuclear actin cap (Gay et al., 2011a). In mesenchymal cells, the actin cap functions in cellular mechanosensation and mechanotransduction, providing a mechanism to transmit extracellular and cytoskeletal forces to the nucleus to coordinate nuclear migration and anchorage, cell migration and modulate gene transcription (Chambliss et al., 2013; Kim et al., 2013).

In polarized epithelial NMuMG cells, a cell line derived from normal mouse mammary epithelial cells, refilin-filamin complexes are formed upon stimulation of the cells with TGF- $\beta$, which triggers EMT (Gay et al., 2011a). EMT is a process by which epithelial cells lose their cell polarity and cell-cell adhesion, and gain migratory and invasive properties. The EMT has fundamental roles in embryo morphogenesis and organ development (Bryant and Mostov, 2008; Lamouille et al., 2014). EMT is not a single event; rather, it is a series of coordinated changes orchestrated by cycles of F-actin remodeling and nuclear transcriptional activities (Haynes et al., 2011; Lamouille et al., 2014; Morris and Machesky, 2015). In polarized NMuMG cells maintained in the absence of TGF- $\beta$, refilins are undetectable, whereas FLNA localizes diffusely on the apical surface (Gay et al., 2011a). In polarized epithelial cells, F-actin is predominantly organized in a cortical belt formed of thin filaments that are tightly associated with cell-cell adhesion sites (Furukawa et al., 2017; Gay et al., 2011a). Upon stimulation of the cells with TGF- $\beta$, refilin-B is stabilized and colocalizes with filamin on parallel actin bundles that form at the apical surface above the nucleus at sites of cell-cell contacts (Fig. 3A; see also Gay et al., $2011 \mathrm{a}, \mathrm{b})$. Here, refilin-B stabilization precedes the downregulation of the tight junction protein ZO1 (J.B., unpublished observation) and correlates with the reorganization of the actin belt (Gay et al., 2011a,b). In contrast to FLNA, which is uniformly distributed over the entire length of the actin bundles, refilin-B immunoreactivity is concentrated on actin bundles at cell-cell boundaries (Fig. 3A). This polarized distribution suggests that refilin-B is required to trigger filamin-dependent actin bundle formation, but it is probably not essential for actin bundle elongation. In a NMuMG cell line stably expressing refilin-B shRNA, downregulation of refilin-B expression is associated with diffuse FLNA staining, a disorganized apical actin network, and a delay in nuclear and cell shape remodeling in response to TGF- $\beta$ stimulation (Gay et al., 2011a). These results identified refilin-B as an early downstream effector protein in TGF$\beta$ signaling and a key regulatory protein for $\mathrm{F}$-actin remodeling and the mechanosensory functions of filamin in the early steps of EMT. The downregulation of refilin-B was, however, not sufficient to fully inhibit EMT. It is possible that either refilin-A or other actin regulators and actin binding proteins, such as members of the ezrin/ radixin/moesin (ERM) family (Haynes et al., 2011), compensate for the absence of refilin-B and contribute to the completion of actin filament remodeling during EMT in the NMuMG cell line.

\section{Role of refilins in filamin-mediated mechanosensing in tissue development}

In mice, disruption of Flnb leads to abnormal differentiation of intervertebral disk chondrocytes into hypertrophic chondrocytes, resulting in ectopic bone formation and intervertebral fusions. This phenotype, which is coincident with upregulated TGF- $\beta$ signaling via Smad2 and Smad3 (Zieba et al., 2016), can be rescued by inactivating the intracellular signaling of TGF $\beta$ that is mediated by Smad3 and Runx2 (Zheng et al., 2007), suggesting that $F \ln b$ exerts a repressive function on TGF- $\beta$-Smad signaling in chondrocyte differentiation and skeletal development (Ferguson et al., 2000; Zieba et al., 2016). At a molecular level, the C-terminal Ig21 of FLNB interacts with Smad proteins and prevents the nuclear translocation and nuclear accumulation of Smad1-Smad3-Smad5 complexes (Sasaki et al., 2001; Zheng et al., 2007; Zieba et al., 2016). In the $F \ln b^{-/-}$mouse, the non-sequestered Smad3 translocates into the nucleus, where it may form a complex with the transcriptional repressor histone deacetylase 4 (HDAC4) and so disrupt the repressive HDAC4-Smad3-Runx2 complex, resulting in the improper activation of Runx2 (Zheng et al., 2007); this, in turn, initiates a cascade of cell fate changes, resulting in endochondral-like ossification of the spine (Zieba et al., 2016). The downstream effectors of TGF- $\beta$ signaling affected by disruption of $F \ln b$ in chondrocyte differentiation are similar to those involved in TGF- $\beta$ signaling in EMT during embryonic development, including nuclear accumulation of Smad proteins (Lamouille et al., 2014) and activation of Runx2 (Tavares et al., 2018). Therefore, common signaling pathways that integrate the mechanosensory and signaling functions of filamins are likely to exist in both EMT and chondroprogenitor differentiation. The fact that both refilin and filamin are implicated in EMT, together with observations pointing to a functional link between refilin and FLNB in cartilaginous skeletal development in mice (Mizuhashi et al., 2014), add further support to this hypothesis.

Based on this, we propose a model of how refilins might modulate mechanosensory and signaling functions of filamins in EMT and chondrocyte differentiation in response to TGF- $\beta$ stimulation (Fig. 3B). In unstimulated cells, filamins crosslink actin filaments into orthogonal networks in the cellular cortical cytoplasm, participate in the anchoring of membrane proteins to the actin cytoskeleton (Calderwood et al., 2001; Nakamura et al., 2007, 2011; Razinia et al., 2012) and contribute to organizing cell-cell junctions (Feng et al., 2006) (Fig. 3B, left panel). TGF- $\beta$ stimulation enables refilin binding to filamin and refilin stabilization. The binding of refilin to filamin changes the actin-branching properties of filamin to favor the formation of apical actin bundles (Fig. 1B), with subsequent disorganization of the cortical actin meshwork, cell junction dissolution and a change in nuclear shape (Fig. 3B, right panel). In vertebrates, intercellular adhesion has an important role in cell fate decisions (Alimperti and Andreadis, 2015), in particular in bone development and homeostasis (Marie et al., 2014). Hence, DKO of both refilin genes or deletion of both FLNB alleles could lead to a misregulation of the spatiotemporal actin cytoskeleton dynamics and cell junction remodeling with similar consequences for chondroprogenitor cell differentiation, leading to skeletal developmental defects (Fig. 3C,D). Our model implies that the actin-bundling function of FLNB is complementary to that of the nuclear Smad signaling that is associated with cell fate changes and 
A

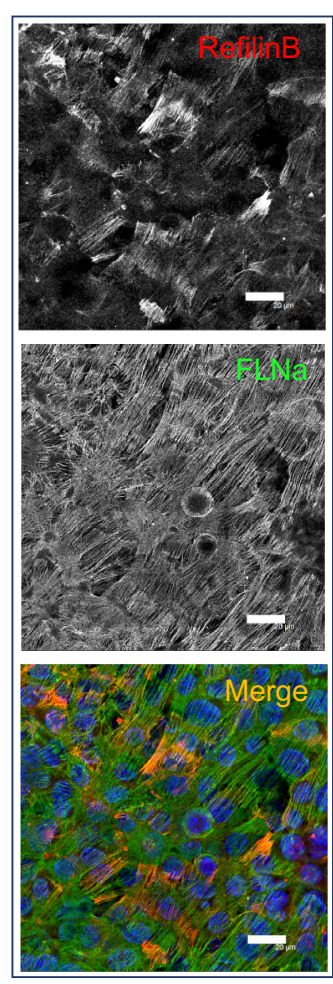

B FLNB+/+; Rfln+/+
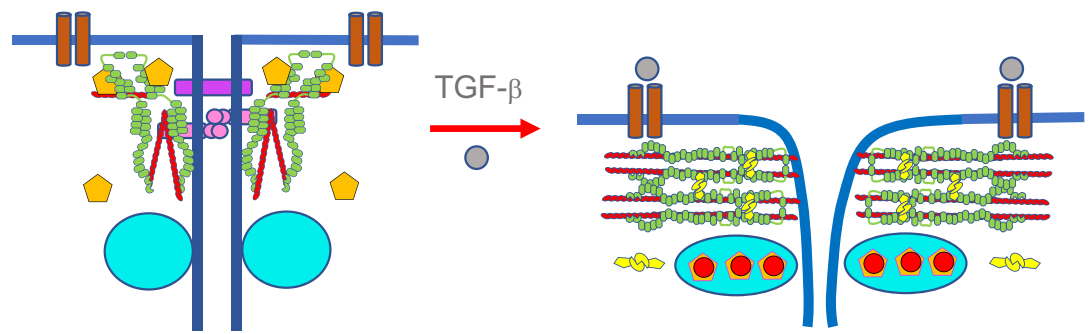

Migration

Differentiation

C FLNB-1-; Rfln+/+
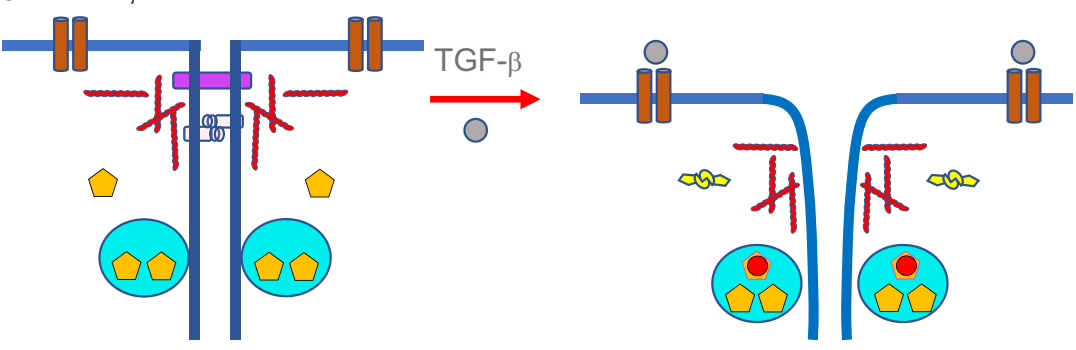

Impaired

migration and differentiation

D FLNB+/+; Rfln-/-
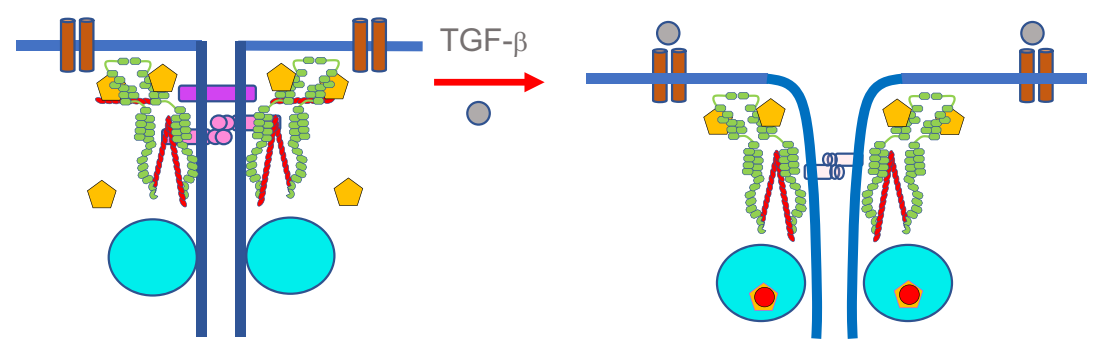

Impaired

migration and

differentiation

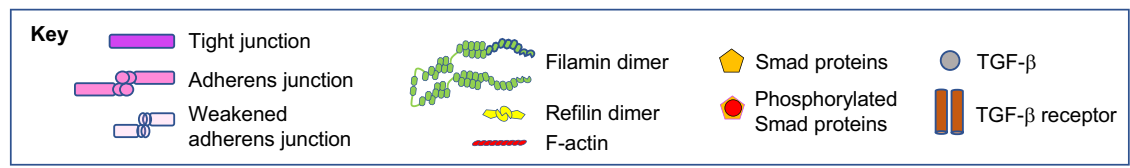

Fig. 3. Proposed molecular mechanisms of refilin-dependent filamin regulation by TGF- $\beta$. (A) Refilin-B colocalizes with FLNA on F-actin bundles on the apical surface of the epithelial NMuMG cells in early stage of EMT. NMuMG cells grown to confluence were stimulated with TGF- $\beta$ (2 ng/ml) for 20 h, fixed with methanol and double immunostained with affinity purified guinea pig anti-refilin-B antibody (red) and mouse anti-FLNA antibody (green). Nuclei were counterstained with Hoechst (blue). Scale bar: $20 \mu \mathrm{m}$. (B-D). TGF- $\beta$ signaling pathways regulate cell fate during EMT and chondroprogenitor differentiation. The refilin-filamin axis plays a central role in spatiotemporal regulation of the actin-cytoskeleton during these cell transitions. (B) In non-stimulated cells (left panel), filamin cross-links actin filaments, anchors actin to the plasma membrane and connects membrane molecules to cytoskeletal complexes to stabilize cell junctions (Feng et al., 2006). The C-terminal IgFLN21 domain of FLNB recruits Smad2 and/or Smad3 and prevents it from being phosphorylated (Sasaki et al., 2001; Zheng et al., 2007). Binding of TGF- $\beta$ to its cell surface receptor activates multiple downstream signaling pathways. Among these is the stabilization of the refilin, which bind to filamin. The refilin-filamin complex promotes formation of a new perinuclear actin network that accompanies nuclear shape changes and cell junctions dissolution (see A) (Gay et al., 2011a). Through binding to the IgFLN21 domain of FLNB, refilins might also compete with and release Smad2 and Smad3 into the cytoplasm. As binding of TGF- $\beta$ enables its receptor-associated kinase to phosphorylate Smad2 and Smad3 (Schmierer and Hill, 2005 ), these accumulate into the nucleus where they interact with transcription factors at regulatory gene sequences to activate or repress transcription (Lamouille et al., 2014). (C) In FLNB-null cells, the loss of FLNB functions weakens adherens junctions and cell-cell interactions (Feng et al., 2006). Smad proteins are released into the cytosol and translocate into the nucleus (Zheng et al., 2007). Upon TGF $\beta$ stimulation, apical actin bundles do not form and the nuclear Smad proteins are less susceptible to phosphorylation by the TGF- $\beta$ receptor-associated kinase. Defective Smad2 phosphorylation in filamin-deficient M2 cells treated with TGF- $\beta$

has been reported (Sasaki et al., 2001). These changes are responsible for a delay in cell shape remodelling, nuclear positioning and possibly defective transcriptional response to TGF- $\beta$ (Sasaki et al., 2001). (D) In refilin DKO cells (Rfln ${ }^{-1-}$ ), phenotypes associated with loss of refilin functions are revealed in response to TGF- $\beta$ stimulation. In the absence of TGF- $\beta$ stimulation, the amount of phosphorylated Smads in the refilin DKO is unaltered when compared to that in wild-type chondrocytes (Mizuhashi et al., 2014). Upon TGF- $\beta$ stimulation, the absence of refilin results in a delay in cell transitions due to misregulation of filamin-mediated actin-bundling and probably a delay in the disolution of adherens cell junctions. The loss of refilin might also reduce the nuclear translocation of filamin-bound Smad proteins with a possibly attenuated transcriptional response to TGF- $\beta$, as in FLNB-null cells. It is expected that in refilin DKO mice (C) and in FLNB-null mice (D), misregulations of actin cytoskeleton dynamics have similar consequences on cell migration, differentiation and developmental defects. See text for further details.

cell differentiation. Smad-dependent TGF- $\beta$ signaling pathways for chondrocyte differentiation and bone formation are recognized to be variable and context-dependent with both synergistic as well as antagonistic activities at play (reviewed in Dexheimer et al., 2016; van der Kraan et al., 2009). Because impaired regulation of chondrocyte migration is also implicated in the accelerated differentiation of these cells in Flnb-null mice (Hu et al., 2014), further studies focusing on investigating the effects of the refilin- 
FLNB complex on chondroprogenitor cell migration should be considered. We hope that the model presented here may serve as a canvas for further experiments to test and validate the different aspects of refilin functions in EMT and chondroprogenitor cell differentiation.

\section{Refilin-enhanced disruption of the mechanosensory properties of mutant FLNB}

Mutations in FLNB that lead to the BD-LS-AO spectrum of conditions concentrate in either the $\mathrm{CH} 2$ domain or IgFLN14-15 (Xu et al., 2017), two regions that function in actin binding (Nakamura et al., 2007). BD, AO and LS-associated mutations enhance the avidity of FLNB-actin binding, suggesting that these diseases might be caused by a disruption of the actin binding and the mechanosensory properties of FLNB (Bicknell et al., 2007; Clark et al., 2009; Daniel et al., 2012; Sawyer et al., 2009), as do analogous mutations in FLNA (Clark et al., 2009) and FLNC (Duff et al., 2011).

When ectopically expressed in cells, a fluorescent EGFP-tagged wild-type FLNB shows either a diffuse cytoplasmic localization with the appearance of a fine meshwork (Daniel et al., 2012) or localizes on actin filaments (Zhao et al., 2016), depending on the cell type. By contrast, BD, AO and LS-associated FLNB mutants are targeted to stress fibers, and also promote the formation of protein aggregates containing F-actin with an intensity that correlates with severity of FLNB-related disease (Daniel et al., 2012). In Rat2 fibroblasts, expression of the mutant protein EGFP-FLNB ${ }^{\text {Glu227Lys }}$ promoted formation of star-shaped actin superstructure (Zhao et al., 2016), whereas in the human HEK 293 cells, the distribution of the EGFP-FLNB ${ }^{\text {Glu227Lys }}$ resembles that of cells transfected with wildtype fusion protein with only minor focal accumulations (Daniel et al., 2012). Hence, cellular factors that modulate the actin-bundling functions of FLNB mutant proteins exist. Co-transfection of BD, AO and LS-associated FLNB mutants with refilin-B in the human U373 cells, which lack endogenous refilin, enhanced the number and size of star-shaped actin superstructures composed of a core of a thick density enriched in F-actin bundles (J.B., Z.A.J. and S.P.R., unpublished data). Similar actin superstructures were previously observed in cells manipulated to increase actin bundles (Mejillano et al., 2004) and in cells characterized by deficient refilin degradation pathways (Gay et al., 2016, 2011a). We would like to suggest that BD, $\mathrm{AO}$ and LS-associated mutations and refilin binding increase the Factin avidity of FLNB mutants through synergistic mechanisms. FLNB mutations increase the actin binding of the N-terminal actinbinding domain to promote or stabilize F-actin bundles (Daniel et al., 2012; Zhao et al., 2016), whereas refilins might synergize the actin binding through activation of the cryptic actin-binding domain localized in the central part of the protein to stabilize actin bundles and to further cross-link F-actin bundles (Fig. 1B). Further studies should explore the possibility for cooperation between refilins and FLNB mutant proteins to alleviate loss of the filamin mechanosensory functions and altered spatiotemporal actin dynamic regulation in $\mathrm{BD}, \mathrm{LS}$ and $\mathrm{AO}$ syndromes.

\section{Conclusions}

Various higher-order networks of actin filaments and their spatiotemporal modulation control cell fate specification and differentiation. The refilins represent the first family of short-lived F-actin regulators that exert a spatiotemporal control on the F-actin cytoskeleton during fundamental embryonic developmental processes. In cells, refilins bind to the actin-binding protein filamin and promote conformational changes that modulate the actin crosslinking and mechanosensing properties of filamin. These changes impact on cell junction organization, cell migration and synergize with the activation of nuclear transcriptional programs. The effect of refilin on the functions of filamin with regard to actin binding and mechanosensing regulation likely occur in competition with other cellular regulators, either actin-assembly factors (Davidson and Wood, 2016; Rotty and Bear, 2014) or signaling proteins such as Smad proteins. Such a competition with other filamin-interacting proteins could be modulated spatially and temporally within the cell through the transcriptional regulation of refilin production and its degradation mechanisms. It is likely that there are other short-lived regulators for the spatiotemporal regulation of actin dynamics that remain to be identified. Finally, the possibility of a synergy between refilins and filamin mutant proteins to stabilize actin bundles opens new avenues to understand diseases caused by missense mutations or small in-frame deletions or insertions in filamin genes (FLNA, FLNB and FLNC). Further studies should explore the synergy between refilin and filamin mutants in the development of filamin-related syndromes.

\section{Acknowledgements}

We thank all the researchers that contributed to the work on refilin proteins in our teams, Olivia Gay, Alexandre Deshiere, Benoit Gilquin, Nicole Assard, Nathalie Bertacchil and Rosannah McCartney. We also thank Justine Jorge and Clemence Raut for stimulating discussion.

\section{Competing interests}

The authors declare no competing or financial interests.

\section{Funding}

S.P.R. is supported by funding from the Health Research Council of New Zealand The Marsden Fund and Curekids New Zealand

\section{Supplementary information}

Supplementary information available online at

http://jcs.biologists.org/lookup/doi/10.1242/jcs.213959.supplemental

\section{References}

Alimperti, S. and Andreadis, S. T. (2015). CDH2 and CDH11 act as regulators of stem cell fate decisions. Stem Cell Res. 14, 270-282.

Ang, X. L. and Wade Harper, J. (2005). SCF-mediated protein degradation and cell cycle control. Oncogene 24, 2860-2870.

Baldassarre, M., Razinia, Z., Burande, C. F., Lamsoul, I., Lutz, P. G. and Calderwood, D. A. (2009). Filamins regulate cell spreading and initiation of cell migration. PLOS ONE 4, e7830.

Bicknell, L. S., Farrington-Rock, C., Shafeghati, Y., Rump, P., Alanay, Y., Alembik, Y., Al-Madani, N., Firth, H., Karimi-Nejad, M. H., Kim, C. A. et al. (2007). A molecular and clinical study of Larsen syndrome caused by mutations in FLNB. J. Med. Genet. 44, 89-98.

Bryant, D. M. and Mostov, K. E. (2008). From cells to organs: building polarized tissue. Nat. Rev. Mol. Cell Biol. 9, 887-901.

Calderwood, D. A., Huttenlocher, A., Kiosses, W. B., Rose, D. M., Woodside, D. G., Schwartz, M. A. and Ginsberg, M. H. (2001). Increased filamin binding to beta-integrin cytoplasmic domains inhibits cell migration. Nat. Cell Biol. 3 1060-1068.

Chambliss, A. B., Khatau, S. B., Erdenberger, N., Robinson, D. K., Hodzic, D. Longmore, G. D. and Wirtz, D. (2013). The LINC-anchored actin cap connects the extracellular milieu to the nucleus for ultrafast mechanotransduction Sci. Rep. 3, 1087.

Clark, A. R., Sawyer, G. M., Robertson, S. P. and Sutherland-Smith, A. J. (2009) Skeletal dysplasias due to filamin A mutations result from a gain-of-function mechanism distinct from allelic neurological disorders. Hum. Mol. Genet. 18, 4791-4800.

Dalkilic, I., Schienda, J., Thompson, T. G. and Kunkel, L. M. (2006). Loss of FilaminC (FLNc) results in severe defects in myogenesis and myotube structure. Mol. Cell. Biol. 26, 6522-6534.

Daniel, P. B., Morgan, T., Alanay, Y., Bijlsma, E., Cho, T.-J., Cole, T., Collins, F. David, A., Devriendt, K., Faivre, L. et al. (2012). Disease-associated mutations in the actin-binding domain of filamin B cause cytoplasmic focal accumulations correlating with disease severity. Hum. Mutat. 33, 665-673.

Davidson, A. J. and Wood, W. (2016). Unravelling the actin cytoskeleton: a new competitive edge? Trends Cell Biol. 26, 569-576. 
DeMaso, C. R., Kovacevic, I., Uzun, A. and Cram, E. J. (2011). Structural and functional evaluation of $\mathrm{C}$. elegans filamins FLN-1 and FLN-2. PLoS ONE 6, e22428.

Dexheimer, V., Gabler, J., Bomans, K., Sims, T., Omlor, G. and Richter, W. (2016). Differential expression of TGF-beta superfamily members and role of Smad1/5/9-signalling in chondral versus endochondral chondrocyte differentiation. Sci. Rep. 6, 36655

Duff, R. M., Tay, V., Hackman, P., Ravenscroft, G., McLean, C., Kennedy, P., Steinbach, A., Schöffler, W., van der Ven, P. F. M., Fürst, D. O. et al. (2011) Mutations in the $\mathrm{N}$-terminal actin-binding domain of filamin $\mathrm{C}$ cause a distal myopathy. Am. J. Hum. Genet. 88, 729-740.

Dyson, H. J. and Wright, P. E. (2005). Intrinsically unstructured proteins and their functions. Nat. Rev. Mol. Cell Biol. 6, 197-208.

Ehrlicher, A. J., Nakamura, F., Hartwig, J. H., Weitz, D. A. and Stossel, T. P. (2011). Mechanical strain in actin networks regulates FilGAP and integrin binding to filamin A. Nature 478, 260-263.

Farrington-Rock, C., Kirilova, V., Dillard-Telm, L., Borowsky, A. D., Chalk, S., Rock, M. J., Cohn, D. H. and Krakow, D. (2008). Disruption of the Flnb gene in mice phenocopies the human disease spondylocarpotarsal synostosis syndrome. Hum. Mol. Genet. 17, 631-641.

Fendri, K., Patten, S. A., Kaufman, G. N., Zaouter, C., Parent, S., Grimard, G., Edery, P. and Moldovan, F. (2013). Microarray expression profiling identifies genes with altered expression in Adolescent Idiopathic Scoliosis. Eur. Spine J. 22 1300-1311.

Feng, Y., Chen, M. H., Moskowitz, I. P., Mendonza, A. M., Vidali, L., Nakamura, F., Kwiatkowski, D. J. and Walsh, C. A. (2006). Filamin A (FLNA) is required fo cell-cell contact in vascular development and cardiac morphogenesis. Proc. Natl. Acad. Sci. USA 103, 19836-19841.

Ferguson, C. M., Schwarz, E. M., Reynolds, P. R., Puzas, J. E., Rosier, R. N. and O'Keefe, R. J. (2000). Smad2 and 3 mediate transforming growth factor-beta1induced inhibition of chondrocyte maturation. Endocrinology 141, 4728-4735.

Fortmann, K. T., Lewis, R. D., Ngo, K. A., Fagerlund, R. and Hoffmann, A. (2015) A regulated, ubiquitin-independent Degron in IkappaBalpha. J. Mol. Biol. 427 $2748-2756$

Fürst, D. O., Goldfarb, L. G., Kley, R. A., Vorgerd, M., Olivé, M. and van der Ven, P. F. (2013). Filamin C-related myopathies: pathology and mechanisms. Acta Neuropathol. 125, 33-46.

Furukawa, K. T., Yamashita, K., Sakurai, N. and Ohno, S. (2017). The epithelial circumferential actin belt regulates YAP/TAZ through nucleocytoplasmic shuttling of merlin. Cell Rep. 20, 1435-1447.

Gay, O., Gilquin, B., Nakamura, F., Jenkins, Z. A., McCartney, R., Krakow, D. Deshiere, A., Assard, N., Hartwig, J. H., Robertson, S. P. et al. (2011a) RefilinB (FAM101B) targets filamin A to organize perinuclear actin networks and regulates nuclear shape. Proc. Natl. Acad. Sci. USA 108, 11464-11469.

Gay, O., Gilquin, B., Pitaval, A. and Baudier, J. (2011b). Refilins: a link between perinuclear actin bundle dynamics and mechanosensing signaling Bioarchitecture 1, 245-249.

Gay, O., Nakamura, F. and Baudier, J. (2011c). Refilin holds the cap. Commun Integr. Biol. 4, 791-795.

Gay, O., Gilquin, B., Assard, N., Stuelsatz, P., Delphin, C., Lachuer, J., Gidrol, X. and Baudier, J. (2016). Refilins are short-lived Actin-bundling proteins that regulate lamellipodium protrusion dynamics. Biol. Open 5, 1351-1361.

Gordon, C. A., Gong, X., Ganesh, D. and Brooks, J. D. (2017). NUSAP1 promotes invasion and metastasis of prostate cancer. Oncotarget 8, 29935-29950.

Gsponer, J., Futschik, M. E., Teichmann, S. A. and Babu, M. M. (2008). Tight regulation of unstructured proteins: from transcript synthesis to protein degradation. Science 322, 1365-1368.

Haynes, J., Srivastava, J., Madson, N., Wittmann, T. and Barber, D. L. (2011) Dynamic actin remodeling during epithelial-mesenchymal transition depends on increased moesin expression. Mol. Biol. Cell 22, 4750-4764.

Hirano, M., Murata, T., Furushima, K., Kiyonari, H., Nakamura, M., Suda, Y. and Aizawa, S. (2005). cfm is a novel gene uniquely expressed in developing forebrain and midbrain, but its null mutant exhibits no obvious phenotype. Gene Expr. Patterns 5, 439-444.

Hu, J., Lu, J., Lian, G., Ferland, R. J., Dettenhofer, M. and Sheen, V. L. (2014) Formin 1 and filamin B physically interact to coordinate chondrocyte proliferation and differentiation in the growth plate. Hum. Mol. Genet. 23, 4663-4673.

Huang, W., Lv, X., Liu, C., Zha, Z., Zhang, H., Jiang, Y., Xiong, Y., Lei, Q.-Y. and Guan, K.-L. (2012). The N-terminal phosphodegron targets TAZ/WWTR1 protein for SCFbeta-TrCP-dependent degradation in response to phosphatidylinositol 3 kinase inhibition. J. Biol. Chem. 287, 26245-26253.

Huelsmann, S., Rintanen, N., Sethi, R., Brown, N. H. and Ylänne, J. (2016). Evidence for the mechanosensor function of filamin in tissue development. Sci. Rep. 6, 32798

Janoštiak, R., Pataki, A. C., Brábek, J. and Rösel, D. (2014). Mechanosensors in integrin signaling: the emerging role of p130Cas. Eur. J. Cell Biol. 93, 445-454.

Kanemori, Y., Uto, K. and Sagata, N. (2005). Beta-TrCP recognizes a previously undescribed nonphosphorylated destruction motif in Cdc25A and Cdc25B phosphatases. Proc. Natl. Acad. Sci. USA 102, 6279-6284.
Kesner, B. A., Milgram, S. L., Temple, B. R. S. and Dokholyan, N. V. (2010) Isoform divergence of the filamin family of proteins. Mol. Biol. Evol. 27, 283-295 Kim, D.-H., Chambliss, A. B. and Wirtz, D. (2013). The multi-faceted role of the actin cap in cellular mechanosensation and mechanotransduction. Soft Mat. $\mathbf{9}$ 5516-5523.

Kovacevic, I. and Cram, E. J. (2010). FLN-1/filamin is required for maintenance of actin and exit of fertilized oocytes from the spermatheca in C. elegans. Dev. Biol. $347,247-257$

Krakow, D., Robertson, S. P., King, L. M., Morgan, T., Sebald, E. T., Bertolotto, C., Wachsmann-Hogiu, S., Acuna, D., Shapiro, S. S., Takafuta, T. et al. (2004). Mutations in the gene encoding filamin B disrupt vertebral segmentation, joint formation and skeletogenesis. Nat. Genet. 36, 405-410.

Kueh, H. Y., Brieher, W. M. and Mitchison, T. J. (2008). Dynamic stabilization of actin filaments. Proc. Natl. Acad. Sci. USA 105, 16531-16536.

Lamouille, S., Xu, J. and Derynck, R. (2014). Molecular mechanisms of epithelialmesenchymal transition. Nat. Rev. Mol. Cell Biol. 15, 178-196.

Light, S., Sagit, R., Ithychanda, S. S., Qin, J. and Elofsson, A. (2012). The evolution of filamin-a protein domain repeat perspective. J. Struct. Biol. 179, 289-298

Luo, T., Mohan, K., Iglesias, P. A. and Robinson, D. N. (2013). Molecular mechanisms of cellular mechanosensing. Nat. Mater. 12, 1064-1071.

Marie, P. J., Haÿ, E. and Saidak, Z. (2014). Integrin and cadherin signaling in bone: role and potential therapeutic targets. Trends Endocrinol. Metab. 25, 567-575.

Mejillano, M. R., Kojima, S., Applewhite, D. A., Gertler, F. B., Svitkina, T. M. and Borisy, G. G. (2004). Lamellipodial versus filopodial mode of the actin nanomachinery: pivotal role of the filament barbed end. Cell 118, 363-373.

Mishra, D. K., Creighton, C. J., Zhang, Y., Chen, F., Thrall, M. J. and Kim, M. P. (2015). Ex vivo four-dimensional lung cancer model mimics metastasis. Ann. Thorac. Surg. 99, 1149-1156.

Mizuhashi, K., Kanamoto, T., Moriishi, T., Muranishi, Y., Miyazaki, T., Terada, K. Omori, Y., Ito, M., Komori, T. and Furukawa, T. (2014). Filamin-interacting proteins, Cfm1 and Cfm2, are essential for the formation of cartilaginous skeletal elements. Hum. Mol. Genet. 23, 2953-2967.

Morris, H. T. and Machesky, L. M. (2015). Actin cytoskeletal control during epithelial to mesenchymal transition: focus on the pancreas and intestinal tract. Br. J. Cancer 112, 613-620.

Nag, S., Larsson, M., Robinson, R. C. and Burtnick, L. D. (2013). Gelsolin: the tail of a molecular gymnast. Cytoskeleton (Hoboken) 70, 360-384.

Nakamura, F., Osborn, T. M., Hartemink, C. A., Hartwig, J. H. and Stossel, T. P. (2007). Structural basis of filamin A functions. J. Cell Biol. 179, 1011-1025.

Nakamura, F., Stossel, T. P. and Hartwig, J. H. (2011). The filamins: organizers of cell structure and function. Cell Adh. Migr. 5, 160-169.

Ohta, Y., Stossel, T. P. and Hartwig, J. H. (1991). Ligand-sensitive binding of actinbinding protein to immunoglobulin G Fc receptor I (Fc gamma RI). Cell 67 275-282.

Pentikäinen, U. and Ylänne, J. (2009). The regulation mechanism for the autoinhibition of binding of human filamin A to integrin. J. Mol. Biol. 393, 644-657.

Razinia, Z., Mäkelä, T., Ylänne, J. and Calderwood, D. A. (2012). Filamins in mechanosensing and signaling. Annu. Rev. Biophys. 41, 227-246.

Risolino, M., Mandia, N., lavarone, F., Dardaei, L., Longobardi, E., Fernandez, S., Talotta, F., Bianchi, F., Pisati, F., Spaggiari, L. et al. (2014). Transcription factor PREP1 induces EMT and metastasis by controlling the TGF-beta-SMAD3 pathway in non-small cell lung adenocarcinoma. Proc. Natl. Acad. Sci. USA 111 E3775-E3784.

Robertson, S. P. (2005). Filamin A: phenotypic diversity. Curr. Opin. Genet. Dev 15, 301-307.

Robertson, S. P., Twigg, S. R. F., Sutherland-Smith, A. J., Biancalana, V. Gorlin, R. J., Horn, D., Kenwrick, S. J., Kim, C. A., Morava, E., Newbury-Ecob, R. et al. (2003). Localized mutations in the gene encoding the cytoskeletal protein filamin A cause diverse malformations in humans. Nat. Genet. 33, 487-491.

Robertson, S. P., Jenkins, Z. A., Morgan, T., Adès, L., Aftimos, S., Boute, O., Fiskerstrand, T., Garcia-Miñaur, S., Grix, A., Green, A. et al. (2006) Frontometaphyseal dysplasia: mutations in FLNA and phenotypic diversity. Am. J. Med. Genet. A 140A, 1726-1736.

Rognoni, L., Stigler, J., Pelz, B., Ylanne, J. and Rief, M. (2012). Dynamic force sensing of filamin revealed in single-molecule experiments. Proc. Natl. Acad. Sci. USA 109, 19679-19684.

Rotty, J. D. and Bear, J. E. (2014). Competition and collaboration between differen actin assembly pathways allows for homeostatic control of the actin cytoskeleton. Bioarchitecture 5, 27-34.

Sasaki, A., Masuda, Y., Ohta, Y., Ikeda, K. and Watanabe, K. (2001). Filamin associates with Smads and regulates transforming growth factor-beta signaling J. Biol. Chem. 276, 17871-17877.

Sawyer, G. M., Clark, A. R., Robertson, S. P. and Sutherland-Smith, A. J. (2009) Disease-associated substitutions in the filamin B actin binding domain confer enhanced actin binding affinity in the absence of major structural disturbance: insights from the crystal structures of filamin B actin binding domains. J. Mol. Biol. $390,1030-1047$ 
Schmierer, B. and Hill, C. S. (2005). Kinetic analysis of Smad nucleocytoplasmic shuttling reveals a mechanism for transforming growth factor beta-dependent nuclear accumulation of Smads. Mol. Cell. Biol. 25, 9845-9858.

Schoeler, C., Malinowska, K. H., Bernardi, R. C., Milles, L. F., Jobst, M. A., Durner, E., Ott, W., Fried, D. B., Bayer, E. A., Schulten, K. et al. (2014) Ultrastable cellulosome-adhesion complex tightens under load. Nat. Commun. 5, 5635.

Seppälä, J., Bernardi, R. C., Haataja, T. J. K., Hellman, M., Pentikainen, O. T., Schulten, K., Permi, P., Ylanne, J. and Pentikäinen, U. (2017). Skeletal dysplasia mutations effect on human filamins' structure and mechanosensing. Sci. Rep. 7, 4218.

Sheen, V. L., Feng, Y., Graham, D., Takafuta, T., Shapiro, S. S. and Walsh, C. A. (2002). Filamin A and Filamin B are co-expressed within neurons during periods of neuronal migration and can physically interact. Hum. Mol. Genet. 11, 2845-2854

Sokol, N. S. and Cooley, L. (1999). Drosophila filamin encoded by the cheerio locus is a component of ovarian ring canals. Curr. Biol. 9, 1221-1230.

Stossel, T. P., Condeelis, J., Cooley, L., Hartwig, J. H., Noegel, A., Schleicher, M. and Shapiro, S. S. (2001). Filamins as integrators of cell mechanics and signalling. Nat. Rev. Mol. Cell Biol. 2, 138-145.

Tavares, A. L. P., Brown, J. A., Ulrich, E. C., Dvorak, K. and Runyan, R. B. (2018). Runx2-I is an early regulator of epithelial-mesenchymal cell transition in the chick embryo. Dev. Dyn. 247, 542-554.

Tsuchiya, Y., Morita, T., Kim, M., lemura, S., Natsume, T., Yamamoto, M. and Kobayashi, A. (2011). Dual regulation of the transcriptional activity of Nrf1 by beta-TrCP- and Hrd1-dependent degradation mechanisms. Mol. Cell. Biol. 31, $4500-4512$.

Umeki, N., Nakajima, J., Noguchi, T. Q. P., Tokuraku, K., Nagasaki, A., Ito, K., Hirose, K. and Uyeda, T. Q. P. (2013). Rapid nucleotide exchange renders Asp11 mutant actins resistant to depolymerizing activity of cofilin, leading to dominant toxicity in vivo. J. Biol. Chem. 288, 1739-1749.

van der Kraan, P. M., Blaney Davidson, E. N., Blom, A. and van den Berg, W. B. (2009). TGF-beta signaling in chondrocyte terminal differentiation and osteoarthritis: modulation and integration of signaling pathways through receptor-Smads. Osteoarthritis Cartilage 17, 1539-1545.

Vogel, V., Thomas, W. E., Craig, D. W., Krammer, A. and Baneyx, G. (2001). Structural insights into the mechanical regulation of molecular recognition sites. Trends Biotechnol. 19, 416-423.

Walrafen, P., Verdier, F., Kadri, Z., Chretien, S., Lacombe, C. and Mayeux, P. (2005). Both proteasomes and lysosomes degrade the activated erythropoietin receptor. Blood 105, 600-608.

Xu, Q., Wu, N., Cui, L., Wu, Z. and Qiu, G. (2017). Filamin B: the next hotspot in skeletal research? J. Genet. Genomics 44, 335-342.

Yoshioka, K., Oda, A., Notsu, C., Ohtsuka, T., Kawai, Y., Suzuki, S., Nakamura, T., Mabuchi, Y., Matsuzaki, Y. and Goitsuka, R. (2015). Loss of the Homeodomain transcription factor Prep1 perturbs adult hematopoiesis in the bone marrow. PLOS ONE 10, e0136107.

Zhao, Y., Shapiro, S. S. and Eto, M. (2016). F-actin clustering and cell dysmotility induced by the pathological W148R missense mutation of filamin B at the actinbinding domain. Am. J. Physiol. Cell Physiol. 310, C89-C98.

Zheng, L., Baek, H.-J., Karsenty, G. and Justice, M. J. (2007). Filamin B represses chondrocyte hypertrophy in a Runx2/Smad3-dependent manner. J. Cell Biol. 178, 121-128.

Zhou, X., Tian, F., Sandzen, J., Cao, R., Flaberg, E., Szekely, L., Cao, Y., Ohlsson, C., Bergo, M. O., Boren, J. et al. (2007). Filamin B deficiency in mice results in skeletal malformations and impaired microvascular development. Proc. Natl. Acad. Sci. USA 104, 3919-3924.

Zhou, A.-X., Hartwig, J. H. and Akyürek, L. M. (2010). Filamins in cell signaling, transcription and organ development. Trends Cell Biol. 20, 113-123.

Zieba, J., Forlenza, K. N., Khatra, J. S., Sarukhanov, A., Duran, I., Rigueur, D., Lyons, K. M., Cohn, D. H., Merrill, A. E. and Krakow, D. (2016). TGFbeta and BMP dependent cell fate changes due to loss of filamin B produces disc degeneration and progressive vertebral fusions. PLoS Genet. 12, e1005936. 